\title{
BMJ Open Rationale and design of a single-blind, randomised controlled trial of exercise training for managing learning and memory impairment in persons with multiple sclerosis
}

\author{
Brian M Sandroff, ${ }^{1}$ Robert W Motl, ${ }^{1}$ Marcus Bamman, ${ }^{2,3}$ Gary R Cutter, ${ }^{4}$ \\ Mark Bolding, ${ }^{5}$ John R Rinker, ${ }^{6}$ Glenn R Wylie, ${ }^{7}$ Helen Genova, ${ }^{7}$ John DeLuca ${ }^{7}$
}

To cite: Sandroff BM, Motl RW, Bamman M, et al. Rationale and design of a single-blind, randomised controlled trial of exercise training for managing learning and memory impairment in persons with multiple sclerosis. BMJ Open 2018;8:e023231. doi:10.1136/ bmjopen-2018-023231

- Prepublication history and additional material for this paper are available online. To view these files, please visit the journal online (http://dx.doi. org/10.1136/bmjopen-2018023231).

Received 27 March 2018 Revised 24 October 2018 Accepted 26 October 2018

Check for updates

(C) Author(s) (or their employer(s)) 2018. Re-use permitted under CC BY-NC. No commercial re-use. See rights and permissions. Published by BMJ.

For numbered affiliations see end of article.

Correspondence to

Professor Brian M Sandroff; sandroff@uab.edu

\section{ABSTRACT}

Introduction This randomised controlled trial (RCT) examines treadmill walking exercise training effects on learning and memory performance, hippocampal volume, and hippocampal resting-state functional connectivity in persons with multiple sclerosis (MS) who have objective impairments in learning new information.

Methods and analysis Forty fully ambulatory persons with MS who demonstrate objective learning and memory impairments will be randomly assigned into either the intervention or active control study conditions. The intervention condition involves supervised, progressive treadmill walking exercise training three times per week for a 3-month period. The active control condition involves supervised, progressive low-intensity resistive exercise that will be delivered at the same frequency as the intervention condition. The primary outcome will involve composite performance on neuropsychological learning and memory tests, and the secondary outcomes involve MRI measures of hippocampal volume and resting-state functional connectivity administered before and after the 3-month study period. Outcomes will be administered by treatment-blinded assessors using alternate test forms to minimise practice effects, and MRI data processing will be performed by blinded data analysts.

Ethics and dissemination This study has been approved by a university institutional review board. The primary results will be disseminated via peer-reviewed publications and the final data will be made available to third parties in applicable data repositories. If successful, the results from this study will eventually inform subsequent RCTs for developing physical rehabilitation interventions (ie, treadmill walking exercise training) for improving learning and memory and its relationship with hippocampal outcomes in larger samples of cognitively impaired persons with MS. The results from this early-phase RCT will further lay preliminary groundwork for ultimately providing clinicians and patients with guidelines for better using chronic treadmill walking exercise for improving cognition and brain health. This approach is paramount as learning and memory impairment is common, burdensome and poorly managed in MS.

Trial registration number NCT03319771; Pre-results.

\section{Strengths and limitations of this study}

- The current randomised controlled trial applies a systematically developed exercise training intervention for improving learning and memory and hippocampal structure and function among persons with multiple sclerosis (MS) who present with objective learning and memory impairment.

- This study is adequately powered and involves the inclusion of an active control comparison condition (ie, low-intensity resistive exercise) as well as blinded MRI data analysts.

- However, this efficacy study does include a relatively short intervention period (ie, 3 months) and will not examine the sustainability and durability of the intervention effects on cognitive and hippocampal neuroimaging outcomes.

- This study was powered based on pilot data on exercise effects on behavioural measures of learning and memory in persons with MS (ie, the primary study outcomes) and not on pilot data on the secondary outcomes of exercise effects on hippocampal volume and functional connectivity.

- This study involves fully ambulatory persons with MS and will not involve persons with substantial ambulatory disability.

\section{INTRODUCTION}

The impairment of learning and memory, particularly with learning new information, is a common, burdensome and poorly managed manifestation of multiple sclerosis (MS). ${ }^{1}$ Over $50 \%$ of patients with MS demonstrate impaired performance on neuropsychological tests of verbal and/or visuospatial learning and memory, ${ }^{1}$ which is associated with hippocampal lesions, atrophy and altered resting-state functional connectivity based on neuroimaging (ie, MRI/functional MRI [fMRI]) studies. ${ }^{2-4}$ MS-related learning and memory impairment and decline have been 
associated with depression, ${ }^{5}$ unemployment, ${ }^{6}{ }^{7}$ loss of independence and social isolation. ${ }^{8}$ Currently, there are no Food and Drug Administration (FDA)-approved pharmacological treatments (ie, disease-modifying and symptomatic therapies) for learning and memory dysfunction in MS. ${ }^{9}$ Cognitive rehabilitation is currently the best-characterised behavioural approach for improving MS-related learning and memory impairment and is seemingly mediated through changes in brain function (ie, increased hippocampal activation and resting-state functional connectivity $\left.^{10-14}\right)$. However, cognitive rehabilitation is difficult to apply outside the clinical setting and does not generally result in physical health benefits beyond improved cognition. This underscores the consideration of other behavioural approaches for managing learning and memory impairment and its potential association with hippocampally mediated functional brain outcomes in MS that can be easily applied outside the clinical setting and result in many physical health benefits; one such approach includes exercise training. ${ }^{15}$

The consideration of exercise for improving learning and memory in persons with MS is based, in part, on the body of literature in the general population that documents robust, beneficial effects of exercise training on memory and hippocampal structure/function. ${ }^{16}$ There further is a substantial body of animal work that describes upregulation of central neurovascular and vascular trophic factors with several weeks of exercise that accompanies such neuropsychological and brain systems changes. ${ }^{1718}$ By comparison, there have been few well-designed, targeted exercise training randomised controlled trials (RCTs) on learning and memory and hippocampal neuroimaging outcomes in MS, although the data are promising. ${ }^{19}$ For example, one recent cross-sectional study described statistically significant, moderate-sized correlations between cardiorespiratory fitness (ie, a presumed surrogate of aerobic exercise training) and hippocampal volume in 35 persons with MS. ${ }^{20}$ Another noteworthy case study involving two memory-impaired persons with MS reported that 3 months of aerobic exercise training (stationary cycling) resulted in a $>50 \%$ increase in learning and memory performance, $16.5 \%$ increase in hippocampal volume, as well as increased hippocampal resting-state functional connectivity. ${ }^{21}$ By comparison, the non-aerobic exercise condition demonstrated minimal changes in those outcomes. ${ }^{21}$ One systematically developed, ${ }^{22}$ pilot RCT examined the effects of aerobic treadmill walking exercise training compared with a waitlist control condition on learning and memory performance and hippocampal viscoelasticity using non-conventional MRI (ie, magnetic resonance elastography) in eight fully ambulatory persons with MS. ${ }^{23}$ Overall, there were small-to-moderate intervention effects on verbal learning and memory performance $(d=0.34)$, and large intervention effects on hippocampal viscoelastic properties (ie, increased hippocampal shear stiffness) $(d>0.94)$. The change in verbal learning and memory was strongly associated with change in hippocampal viscoelasticity $(r>0.93$, $\mathrm{p}<0.01)$. Collectively, despite experimental design ${ }^{20}$ and sample size ${ }^{2123}$ limitations, such preliminary observations suggest that aerobic exercise training might improve learning and memory through neuroplasticity in MS and warrant the development of an adequately powered, early-phase RCT for examining the effects of aerobic exercise training on learning and memory and hippocampal structure/function in a larger sample of persons with MS.

The present RCT aims to provide the first evidence for treadmill walking exercise training effects on learning and memory, hippocampal volume, and hippocampal resting-state functional connectivity in an adequately powered sample of persons with MS who have objective impairment in learning new information. This will provide the first evidence for exercise training as a possible treatment for MS-related learning and memory impairment (ie, beyond merely improving learning and memory performance), given that previous trials of exercise, cognition and neuroimaging have not recruited persons with objective MS-related learning and memory impairment a priori (eg, ref 23). The current RCT will further provide the first high-quality evidence of potential mechanisms of aerobic exercise-related effects on hippocampal outcomes in MS, whereas previous studies have been limited by experimental design (eg, ref 20) or sample size (eg, refs 21 23). This study will lay the foundation for a definitive phase III RCT by providing effect sizes of treadmill walking exercise training versus an active control condition on the outcomes that can be used in power analyses for determining the appropriate sample size for such a trial. The present study will provide preliminary experimental evidence of the potential mechanisms (ie, improved hippocampal structure and function) of how treadmill walking exercise might improve learning and memory in this population.

\section{METHODS AND ANALYSIS}

\section{Experimental overview and hypotheses}

The study protocol was drafted in accordance with the Standard Protocol Items: Recommendations for Interventional Trials statement. ${ }^{24}$ This study is registered at ClinicalTrials.gov: NCT03319771. All potential protocol modifications will be approved by the University of Alabama at Birmingham Institutional Review Board (UAB IRB) and will be reported at ClinicalTrials.gov. The proposed study, data collection and intervention will take place at UAB in Birmingham, Alabama, USA. This study involves a single-blind, early phase II RCT on the effects of supervised treadmill walking exercise training compared with an active control condition (ie, low-intensity resistive exercise) on learning and memory, hippocampal structure/function, and cardiorespiratory fitness outcomes in 40 fully ambulatory (ie, Expanded Disability Status Scale (EDSS) $\leq 4.0$ ) persons with MS who have impairment in learning new information. Composite performance on neuropsychological tests of learning and memory represents the primary outcome, whereas neuroimaging 
outcomes of hippocampal volume and hippocampal resting-state functional connectivity represent the secondary outcomes. Cardiorespiratory fitness changes will be included as a manipulation check for documenting the success of the intervention.

During baseline, participants will first complete a battery of neuropsychological tests addressing verbal and visuospatial learning and memory; a maximal, graded exercise test on a motor-driven treadmill to measure cardiorespiratory fitness; and an MRI scan for measurement of hippocampal volume and resting-state functional connectivity. Those outcomes will be measured by assessors who are uninvolved in the exercise training (ie, treatment-blinded assessors). After baseline testing, participants will be randomly assigned to either the intervention or active control conditions using concealment. Participants further will be masked to condition (ie, unaware that the treadmill walking exercise training condition represents the intervention condition and the low-intensity resistive exercise condition represents the active control condition).

The intervention condition will include 3 months of supervised, progressive light-intensity, moderate-intensity and vigorous-intensity treadmill walking exercise, and will be based on the American College of Sports Medicine (ACSM) guidelines for exercise prescription ${ }^{25}$ and pilot work. ${ }^{23} 26-28$ The exercise training itself will take place three times per week over 3 months, and will be facilitated by trained exercise leaders. The exercise prescription will initially consist of $15 \mathrm{~min}$ of light-to-moderate intensity treadmill walking exercise and eventually progress to $40 \mathrm{~min}$ of vigorous-intensity exercise by month 3 (table 1). We note that this exercise stimulus is identical to that of our pilot RCT in eight persons with MS. ${ }^{23}$ The active control condition will involve low-intensity resistive exercise that will be delivered using the same frequency and duration of the treadmill walking exercise condition and facilitated by trained exercise leaders. This is a methodological improvement over our pilot RCT that involved a waitlist (ie, passive) control condition. ${ }^{23}$ Regardless of the assigned condition, all participants will be asked to not undertake additional exercise (ie, not to join a gym and begin exercising) over the duration of the study. The cognitive, cardiorespiratory fitness and MRI outcomes will be assessed again following the 3-month study period by treatment-blinded assessors.

The primary hypothesis is that those who undergo treadmill walking exercise training will demonstrate larger improvements in composite learning and memory performance than those who undergo low-intensity resistive exercise. We further hypothesise that those who undergo treadmill walking exercise training will demonstrate greater increases in hippocampal volume and hippocampal resting-state functional connectivity (ie, adaptive increases) and improvements in cardiorespiratory fitness than those in the low-intensity resistive exercise condition. We lastly hypothesise that (1) the treadmill walking exercise-induced improvements in learning and memory will be accounted for by hippocampal volume and hippocampal resting-state functional connectivity (ie, partial mediation); and (2) those with the largest improvements in cardiorespiratory fitness will demonstrate the largest improvements in the primary and secondary endpoints.

\section{Participants}

Sample size

We plan to enrol 40 fully ambulatory persons with MS (ie, 20 per condition) who have impairment in learning new information (see below); this is based on a power analysis and presumed $15 \%$ attrition. The minimal sample size of 34 persons with MS (ie, 17 per condition) was determined based on power analysis using standard assumptions of alpha $(0.05)$ and beta $(0.80)$ for detecting moderate-sized effects (ie, Partial $\eta^{2}=0.06$ ) based on Cohen's guidelines ${ }^{29}$ for a time by condition interaction in mixed-factor analysis of variance (ANOVA) on composite learning and

Table 1 Exercise prescription and progression over the 3-month period for treadmill walking exercise training condition based on pilot work and the ACSM guidelines

\begin{tabular}{|c|c|c|c|c|}
\hline Week & Sessions & Exercise intensity (\%) & $\begin{array}{l}\text { Exercise } \\
\text { duration (min) }\end{array}$ & Training stage \\
\hline \multicolumn{5}{|c|}{ Baseline testing } \\
\hline 2 & $4-6$ & $40-50 \mathrm{VO}_{2} \mathrm{R} / \mathrm{HRR}$ & $20-25$ & Initiation \\
\hline 3 & $7-9$ & $50-60 \mathrm{VO}_{2} \mathrm{R} / \mathrm{HRR}$ & $20-25$ & Improvement \\
\hline $7-8$ & $19-24$ & 60-70 VO $2 \mathrm{R} / \mathrm{HRR}$ & $30-35$ & Improvement \\
\hline $9-10$ & $25-30$ & $70-80 \mathrm{VO}_{2} \mathrm{R} / \mathrm{HRR}$ & $30-35$ & Maintenance \\
\hline $11-12$ & $31-36$ & $70-80 \mathrm{VO}_{2} \mathrm{R} / \mathrm{HRR}$ & $35-40$ & Maintenance \\
\hline
\end{tabular}

ACSM, American College of Sports Medicine; HRR, heart rate reserve; $\mathrm{VO}_{2} \mathrm{R}$, oxygen consumption reserve. 
memory performance. Our previous pilot RCT of treadmill walking exercise training on cognition yielded a large time by condition interaction on composite learning and memory performance (ie, Partial $\eta^{2}=0.11$ ) in fully ambulatory persons with MS. ${ }^{23}$ Another study on aerobic exercise effects on hippocampal outcomes in persons with schizophrenia reported large intervention effects on hippocampal volume (ie, Partial $\eta^{2}=0.50$ ). ${ }^{30}$ Using those effect sizes as a guide, the proposed sample size of 34 and planned sample size of 40 will be more than adequate for detecting moderate or larger effects on learning and memory and hippocampal outcomes in persons with MS. The required sample size of 34 also adequately powers the secondary study hypotheses based on previous cross-sectional data in MS that indicate moderate-sized correlations (ie, $\rho=0.42$ ) between cardiorespiratory fitness and hippocampal volume. ${ }^{20}$

\section{Recruitment}

Subjects will be recruited directly through the UAB Multiple Sclerosis Center, the Alabama-Mississippi Chapter of the National MS Society (NMSS) and our laboratory database of previous participants with MS who have enquired about participating in exercise studies. Advertisements for the study will be distributed through the UAB Multiple Sclerosis Center, facilitators of local MS support groups, MS Connection publications and email distributions. As a backup plan, we may recruit prospective participants with MS through the North American Research Committee on Multiple Sclerosis patient registry or the iConquerMS if enrolment is slow.

\section{Inclusion/Exclusion criteria}

All participants will be between the ages of 18 and 54, have a clinically definite MS diagnosis based on established criteria (ie, ref 31) and be fully ambulatory based on the EDSS $^{32}$ scores between 0 and 4.0. All participants will demonstrate impairment in learning new information based on open-trial Selective Reminding Task (SRT) scores of at least 1.5 SD below the normative score for healthy controls. ${ }^{33}$ Participants will be relapse-free for at least 30 days (ie, relative neurological stability), and will not have a history of schizophrenia, bipolar disorder I or II, or substance-abuse disorders. Participants further will not be taking medications that can affect cognition (eg, antipsychotics, benzodiazepines), and all participants will be on a stable FDA-approved disease-modifying therapy (eg, interferon beta-1a, interferon beta-1b, glatiramer acetate, natalizumab, dimethyl fumarate and so on) regimen for at least 6 months. Participants will be righthanded (to control for organisation of the brain) and will have a low risk for contraindications for maximal exercise testing based on a 'no' response on all items of the Physical Activity Readiness Questionnaire ${ }^{34}$ or a single 'yes' response along with a physician's approval. Participants further will have a low risk for contraindications for MRI based on (1) not having metal (eg, non-MRI compatible aneurysm clips, metal shards in the body or eyes, or recently placed surgical hardware) or electronic (eg, pacemaker, cochlear implant) devices within the body. Lastly, participants will not be meeting public health guidelines for participating in physical activity (ie, at least $150 \mathrm{~min}$ per week of moderate-to-vigorous aerobic activity). This will be based on health contribution scores on the Godin Leisure-Time Exercise Questionnaire (GLTEQ) that fall within the 'insufficiently active' classification (ie, less than 14 arbitrary units on the summed 'strenuous' and 'moderate' sections of the GLTEQ). ${ }^{35}$

\section{Outcome measures}

To minimise threats to internal validity and maximise the rigour and reproducibility of the present RCT, the outcome measures will be assessed by the personnel who are uninvolved with the intervention or control conditions (ie, treatment-blinded assessors). The outcome assessments further will occur at a different UAB laboratory than the intervention or control conditions to prevent possible contamination.

\section{Learning and memory}

Participants will undertake several neuropsychological tests addressing various aspects of learning and memory as the primary study outcomes. Neuropsychological testing will occur in a quiet, sound-dampened room in the Exercise Neuroscience Research Laboratory (ENRL) at UAB. These tests include the California Verbal Learning Test-II $\left(\right.$ CVLT-II ${ }^{36}$ ) and the SRT ${ }^{37}$ as measures of verbal learning and memory, and the Brief Visuospatial Memory Test-Revised (BVMT-R ${ }^{38}$ ) and the 10/36 Spatial Recall Test $\left(\right.$ SPART $\left.^{39}\right)$ as measures of visuospatial learning and memory. These neuropsychological tests have strong psychometric properties (eg, ref 40) and are widely used to document the efficacy of cognitive rehabilitation interventions in persons with MS. ${ }^{12} 4142$

Briefly, the CVLT-II involves the examiner reading a list of 16 words, with four items belonging to four categories (eg, vegetables, animals, furniture, modes of transportation) that are randomly arranged. The list is read aloud five times in the same order, with each word voiced at a rate of approximately one word per second. Participants are instructed to recall as many items as possible, in any order, following each list reading. The primary outcome of the CVLT-II is the total number of correct words identified over the five trials (ie, raw score) ${ }^{36}$ The SRT involves the examiner reading a list of 12 unrelated words. Participants are asked to recall as many words as possible following the presentation of the list. After the first trial, only the words that participants did not recall are given as the new list. However, participants are instructed to recall as many words as possible from the original (ie, entire) list for each of the five total trials. The primary outcome is the total number of correctly recalled words across the five trials. ${ }^{37}$ For both the CVLT-II and SRT, higher scores indicate better verbal learning and memory.

The BVMT-R involves three trials of the examiner presenting a $2 \times 3$ array of abstract geometric figures in 
front of the participant for $10 \mathrm{~s}$. Following this period, the array is removed and participants are required to draw the array as precisely as possible, with the figures in the correct location. Each drawing is scored on a 0-2 scale, based on accurately portraying each figure and its correct location. The primary outcome of the BVMT-R is the total raw score across the three trials, with higher scores indicating better visuospatial memory. ${ }^{38}$ The $10 / 36$ SPART involves three trials of the examiner presenting a $6 \times 6$ checkerboard with 10 pieces positioned in certain locations on the board in front of the participant for $10 \mathrm{~s}$. Following this period, the display is removed, and participants are asked to replicate the design of the checkerboard on a blank grid. This is repeated for two additional trials. The primary outcome of the $10 / 36$ SPART is the total number of correct responses across the three trials. ${ }^{39}$

As the primary study outcome involves composite learning and memory performance, we will first compute $z$-scores that account for age, sex and education based on normative scores per individual learning and memory test (ie, CVLT-II, SRT, BVMT-R and 10/36 SPART) ${ }^{4344} \mathrm{We}$ will then combine the $z$-scores into a composite learning and memory measure (ie, mean of z-scores for CVLTII, SRT, BVMT-R and 10/36 SPART). Given evidence of hippocampal lateralisation of verbal and visuospatial learning and memory in persons with $\mathrm{MS}^{45}{ }^{4}$ we further will examine the effects of the intervention on those constructs separately in exploratory analyses.

\section{Hippocampal volume and resting-state functional connectivity}

Participants will undergo neuroimaging, which will include structural imaging as well as a resting-state scan. The MRI instrument that will be used is an FDA-approved Siemens MAGNETOM Skyra 3T clinical imager housed in the Civitan International Neuroimaging Research Center at UAB. Each scan session will begin with the acquisition of high-resolution T1-weighted axial anatomical images Magnetization Prepared Rapid Acquisition Gradient Echo (MP-RAGE). This three-dimensional isotropic sequence will be acquired sagittally (Repetition time $(\mathrm{TR})=11.6 \mathrm{~ms}$, Echo time $(\mathrm{TE})=4.9 \mathrm{~ms}$, flip angle $=8^{\circ}$, effective Invation time (TI) $=1017.6 \mathrm{~ms}$, $256 \times 256$ matrix, Field of view $(\mathrm{FOV})=300 \mathrm{~mm}$, Number of excitations $(\mathrm{NEX})=1,172$ slices, $1.17 \mathrm{~mm}$ slice thickness, $0 \mathrm{~mm}$ skip). The total imaging time for this sequence is $8 \mathrm{~min} 38 \mathrm{~s}$. In addition, an inversion-recovery sequence will be acquired axially $(\mathrm{TR}=8530 \mathrm{~ms}, \mathrm{TE}=81 \mathrm{~ms}$, flip angle $=180^{\circ}, 256 \times 256$ matrix, FOV $=220 \mathrm{~mm}, \mathrm{NEX}=1,32$ slices, $4 \mathrm{~mm}$ slice thickness, $0 \mathrm{~mm}$ skip). Together, these scans will be used for volumetric analyses and for image segmentation and normalisation of the resting-state fMRI scan. Functional imaging will consist of multislice gradient echo, $\mathrm{T} 2 *$-weighted images acquired with echoplanar imaging methods $(\mathrm{TE}=60 \mathrm{~ms}, \mathrm{TR}=2000 \mathrm{~ms}$, $\mathrm{FOV}=24 \mathrm{~cm}$, flip angle $=90^{\circ}$, slice thickness $=5 \mathrm{~mm}$ contiguous, matrix $=64 \times 64$, inplane resolution $=2.50 \mathrm{~mm}^{2}$ ). In order to provide coverage of the entire brain, a total of 32 images will be acquired in the axial plane. For the resting-state scan, 180 volumes will be acquired. Structural volumes for the hippocampus will be calculated using the FreeSurfer automated brain segmentation software (http://surfer.nmr.mgh.harvard.edu). Preprocessing of the resting-state functional connectivity data will be performed using the Analysis of Functional NeuroImages (AFNI) software (http://afni.nimh.nih. gov/afni/). The first three volumes will be removed in order to control for saturation effects. Preprocessing steps include slice timing correction, realignment to an image exactly half way through the acquisition run using affine transformation, coregistration to the T1 MP-RAGE image for localisation of activated areas, smoothing (6 mm FWHM, full width half maximum) to minimise anatomical differences and increase the signal to noise ratio, scaling each voxel to the grand mean intensity of that voxel (across the acquisition run), high-pass filtering $(150 \mathrm{~s})$, and normalisation using a non-linear approach (3dQwarp) to a standardised T1 template from the Montreal Neurological Institute. In all cases, the data will be checked for excessive motion (a shift of more than $3.5 \mathrm{~mm}$, or $1^{\circ}$ of angular motion) and for spikes (using the root mean squared error [RMSE] of each volume relative to a reference volume [which will be the volume half way through the acquisition run]). Data acquisition runs with excessive motion will be discarded. Individual acquisitions with an RMSE amplitude that exceeds the 75th percentile plus the value of $150 \%$ of the IQR of RMSE for all volumes in a given run will be excluded from further analysis using the 'censorTR' function in 3dDeconvolve. In all cases, the motion parameters from the realignment step will be used as regressors of no interest in the deconvolution, and the residuals will be saved. The residuals for each subject in each group (exercise and control) will then be included in a probabilistic independent components analysis (ICA), using AROMA (ICA-based Automatic Removal Of Motion Artifacts), as implemented in FSL (http://fsl.fmrib.ox.ac.uk/fsl/fslwiki/). The results of the ICA will be spatial maps of the resting-state networks. We will extract the connectivity map of the hippocampal network that will then be used in a dual-regression analysis. ${ }^{46}$ In the first regression (spatial regression), the spatial hippocampal map from the ICA is regressed onto each subject's functional data, resulting in a data set that characterises the temporal dynamics of the hippocampal network. In the second regression (temporal regression), the data set resulting from the spatial regression (the temporal dynamics of the hippocampal network) is regressed onto each subject's functional data. This results in a map for the hippocampal network representing each voxel's connectivity with this network. This map will then be used in the group-level analyses. Importantly, all MRI processing and analyses will be performed by scientists at Kessler Foundation (ie, HG, GRW), who will be blinded to the condition. To our knowledge, this is among the first exercise RCTs in any population to include this additional level of rigour to enhance the proposed trial's reproducibility. 


\section{Cardiorespiratory fitness}

Cardiorespiratory fitness will be measured as peak oxygen consumption $\left(\mathrm{VO}_{2 \text { peak }}\right)$, using a maximal, graded exercise test on a motor-driven treadmill and an open-circuit spirometry system (Parvo Medics TrueOne 2400, Sandy, Utah) for analysing expired gases using a modified Balke protocol in the ENRL at UAB. This protocol was successfully used in our previous small pilot RCT. ${ }^{23}$ This protocol further is commonly used for measuring cardiorespiratory fitness in older adults ${ }^{47}$ and persons with chronic stroke ${ }^{48}$ and is consistent with the ACSM guidelines for exercise testing of patients with MS. ${ }^{25}$ The test will be preceded by a 3 min warm-up. The initial work rate for the exercise test will be at a brisk, but submaximal pace, and the grade will continuously increase at a rate of $2.0 \%$ every 2 min until the participant reaches volitional fatigue. Heart rate (HR) and rating of perceived exertion will be recorded every minute during the test. $\mathrm{VO}_{2 \text { peak }}$ will be expressed in $\mathrm{mL} / \mathrm{kg} / \mathrm{min}$ based on the highest recorded $20 \mathrm{~s}_{2}$ value when two of four criteria are satisfied: (1) $\mathrm{VO}_{2}$ plateau with increasing grade; (2) respiratory exchange ratio $\geq 1.10$; (3) peak HR within 10 beats/min of age-predicted maximum (ie, $1 \mathrm{SD}$ ); or (4) peak rating of perceived exertion $\geq 17$. $^{49}$ The test will be followed by a 3 min cool-down period.

\section{Disability status}

Participants will undergo a neurological examination by a neurologist (JRR) for generating a baseline EDSS score. All participants will be fully ambulatory (ie, EDSS scores $\leq 4.0)$.

\section{Additional neuropsychological tests}

To evaluate the effects of the intervention on other domains of cognition that are commonly impaired in MS, we will apply the Minimal Assessment of Cognitive Function in Multiple Sclerosis (MACFIMS) neuropsychological battery ${ }^{50}$ as exploratory outcomes. In addition to the CVLT-II and BVMT-R, the MACFIMS includes valid and reliable tests of cognitive processing speed, ${ }^{5152}$ executive function, ${ }^{53}$ verbal fluency ${ }^{54}$ and spatial perception. ${ }^{54}$ Further, baseline performance on the MACFIMS neuropsychological battery will serve to characterise the overall baseline cognitive status of the sample. For all neuropsychological tests, including measures of learning and memory, alternate forms will be applied at each testing session (ie, baseline and follow-up) to minimise the effects of practice on cognition.

\section{Additional neuroimaging outcomes}

The MRI protocol further will involve the collection of structural MRI data on T2 lesion volume as a potential covariate of intervention effects, given its association with MS-related cognitive dysfunction. ${ }^{55}$

\section{Intervention condition}

The intervention condition will include 3 months of supervised, progressive light-intensity, moderate-intensity and vigorous-intensity treadmill walking exercise training based on the ACSM guidelines for maximising adaptations with exercise training. This will occur at a laboratory exercise research facility. Exercise intensities will be prescribed based on per cent oxygen consumption reserve $\left(\% \quad \mathrm{VO}_{2} \mathrm{R}\right)$ using values derived from the baseline graded exercise test. We note that HR reserve (HRR) and $\mathrm{VO}_{2} \mathrm{R}$ have a 1:1 relationship. ${ }^{25}$ The exact exercise prescription is presented in table 1 and further represents the identical stimulus that was included in our pilot RCT that demonstrated improvements in learning and memory and hippocampal neuroimaging outcomes. ${ }^{23}$ The exercise progression in terms of duration and intensity will involve three distinct stages: (1) the initiation stage, (2) the improvement stage and (3) the maintenance stage. The initiation stage (weeks 1-2) aims to prepare participants for more intense aerobic exercise (ie, by accumulating small improvements in cardiorespiratory fitness with light-to-moderate intensity exercise) and develop an orthopaedic tolerance for exercise stress. ${ }^{25}$ Following this period, participants will progress to the improvement stage of exercise training. This stage provides a gradual increase in the overall aerobic exercise stimulus (ie, moderate-to-vigorous intensity), whereby participants realise substantial improvements in cardiorespiratory fitness (weeks 3-8). ${ }^{25}$ The final stage of exercise progression is the maintenance stage (ie, vigorous intensity), which aims to maintain the levels of cardiorespiratory fitness that were developed during the improvement stage over the long term (weeks 9-12).${ }^{25}$ Consistent with the ACSM recommendations, the intervention will not involve progression of both intensity and duration in a single exercise session. Such a gradual progression of exercise training is advantageous for deconditioned persons to safely achieve the benefits of aerobic exercise training. ${ }^{25}$

The exercise training itself will be led by certified exercise leaders who are not involved in the collection of outcome assessments. At the outset of each session, participants will be fitted with a Polar Heart Rate Monitor (Oy, Finland), and HR will be monitored continuously throughout each session. Each session will begin with a 5-10 min warm-up, followed by the exercise; the target HRR range associated with the $\mathrm{VO}_{2} \mathrm{R}$ range will be maintained for as long as possible during each exercise period. This will be followed by a $5-10 \mathrm{~min}$ cool-down. Participants will complete an exercise log at the conclusion of each session for better characterising of the experience with the intervention. Log data will include perceived exertion, ${ }^{56}$ well-being, enjoyment and mental/physical fatigue. Throughout each session, we further will collect data on treadmill speed and grade, as well as time spent within the prescribed $\mathrm{VO}_{2} \mathrm{R} / \mathrm{HRR}$ range for improving the rigour and reproducibility of the intervention.

\section{Active control condition}

The active control condition will involve supervised, low-intensity resistive exercise in order to control for the effects of social contact and attention. This condition 


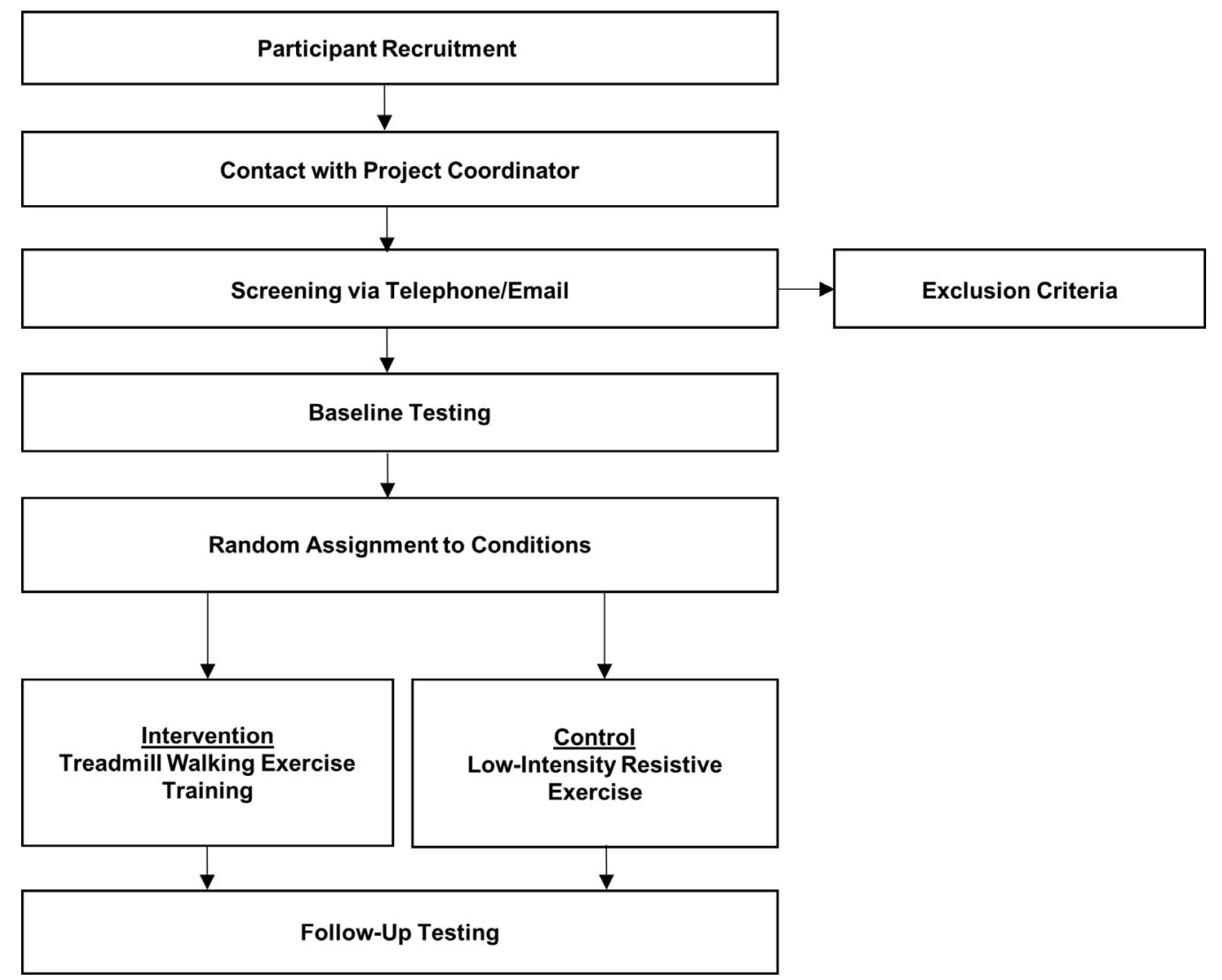

Figure 1 Participant flow through the study.

will take place at a laboratory exercise research facility in a space that is isolated from the intervention condition in order to avoid participant and site contamination. The low-intensity resistive exercise control condition will be delivered using the same frequency and duration of the treadmill walking exercise training condition. The low-intensity resistive exercises will be based on a manual provided by the NMSS ${ }^{57}$ and sessions will be led by certified exercise leaders who are not involved in the collection of outcome assessments. Low-intensity resistive exercises will target the head/neck, shoulder, elbow/forearm, hand/wrist, trunk/hip and ankle/foot. The progression of activities over the 3-month period will involve performing additional exercises and sets, along with using progressively thicker elastic resistance bands (ie, Therabands) that provide minimal resistance. The first 6 weeks of the intervention period will involve performing the activities without resistance. In weeks $7-8$, the extra-thin Theraband (ie, least resistance) will be used to perform the exercises for the upper extremities only. In weeks $9-10$, the thin Theraband will be introduced, and in weeks 11-12 the medium Theraband will be introduced. Such a progression is not expected to induce cardiorespiratory fitness adaptations and is designed to maintain participant interest. Each session is designed to last up to $60 \mathrm{~min}$ in total. Each session will begin with a $5-10 \mathrm{~min}$ warm-up, followed by low-intensity resistive exercise (following the same duration as the treadmill walking exercise training condition) activities and a 5-10 min cool-down. Throughout each session, participants will wear an HR monitor, and we will collect data on HR and perceived exertion ${ }^{56}$ to ensure that this condition occurs at a low intensity. This stimulus has been included as a control comparison condition in a recent exercise training RCT in persons with MS and did not result in cognitive improvements and was well received with no increase in dropout compared with progressive exercise training. ${ }^{58}$

Importantly, to minimise attrition for both the intervention and control conditions, exercise leaders will apply highly developed principles and techniques associated with Social Cognitive Theory ${ }^{59}$ for enhancing participant adherence and compliance throughout each session. Further, regardless of the assigned condition, all participants will be asked to not undertake additional exercise (ie, not to join a gym and begin exercising).

\section{Procedure}

Participant flow through the study is presented in figure 1. Participant recruitment, contact and screening will be undertaken via telephone and/or email by an ENRL project coordinator. If a participant satisfies the initial inclusion/exclusion criteria, the project coordinator will then administer the open-trial SRT via telephone to ensure that all participants have impairments in learning new information; the open-trial SRT has been used as a screening tool for impairments in learning new information in previous memory rehabilitation RCTs in MS. ${ }^{12}$ 
This test involves the project coordinator reading a list of words to the prospective participant over the phone. The participant then repeats as many words as they can from memory back to the project coordinator. This process is repeated until participants can remember the entire list of words. If participants demonstrate scores that are at least 1.5 SD below the normative score for healthy controls, the project coordinator will request contact information from the potential participant's neurologist, whereby they will email or fax a letter asking them to verify a definite MS diagnosis and confirmation that the participant has been on a stable disease-modifying therapy (DMT) regimen for at least 6 months. On receipt of these materials from the participant's neurologist, the project coordinator will schedule the participant for baseline testing. Baseline testing will be led by assessors who are blinded to the condition, and will take place over three non-consecutive days to minimise cognitive and physical fatigue. On the first day, participants will initially provide written informed consent (see online supplementary file 1 for a sample informed consent document) with IRB-approved ENRL personnel, followed by a neurological examination for generation of an EDSS score. Participants will then undertake several neuropsychological tests of learning and memory (ie, CVLT-II, BVMT-R) as part of the full MACFIMS neuropsychological battery, followed by the graded exercise test. The second day of baseline testing will involve the MRI protocol, and the third day of baseline testing will involve undertaking other neuropsychological tests of learning and memory (ie, SRT, 10/36 SPART). Participants will be remunerated $\$ 50$ for completing the baseline assessments. Of note, the separation of learning and memory measures across testing sessions is necessary considering that there might be overlap (interference) between performance on the CVLT-II and SRT (ie, learning and memory tests in the verbal domain) and between the BVMT-R and 10/36 SPART (ie, learning and memory tests in the visuospatial domain), respectively.

After baseline testing, participants will be randomly assigned to either the treadmill walking exercise training or active control conditions using concealment (ie, opaque, sealed envelopes) and computerisation by the study biostatistician (ie, GRC). Participants further will be blinded to the intent of the condition (ie, unaware that the treadmill walking exercise training condition represents the experimental condition and the low-intensity resistive exercise condition represents the active control condition). To do this, the study will be advertised as a comparison of two different physical exercise programmes on memory performance in persons with MS. We note that it is not possible for participants to be blinded to the actual condition (ie, participating in treadmill walking exercise or low-intensity resistive exercise activities).

Participants will undertake the intervention or active control conditions as described above over a 3-month period. Participants will be remunerated $\$ 10$ per treadmill walking exercise/low-intensity resistive exercise visit attended (ie, up to $\$ 360$ total). This remuneration will be disbursed in weekly increments for maximising compliance with this early-stage research. Following the completion of the 3-month study period, participants will again undergo assessments of learning and memory, hippocampal volume and resting-state functional connectivity, and cardiorespiratory fitness using the same procedures as baseline testing (ie, follow-up testing). Follow-up measures, using alternate forms where possible, will be administered by treatment-blinded assessors. Participants will be remunerated $\$ 50$ for completing the follow-up assessments.

\section{Data integrity}

All data will be entered, checked and double-checked by the UAB ENRL personnel under the direct supervision of the ENRL project coordinator and study principal investigator (BMS). All ENRL personnel have undergone extensive training in good clinical practice and laboratory procedures. Given that the current study is not a multisite, National Institutes of Health-defined phase III RCT, we do not include a formal data monitoring committee.

\section{Statistical analysis}

The data analyses will be overseen by a biostatistician (ie, GRC) and follow the intent-to-treat principles (ie, include all persons regardless of adherence and/or compliance). In case of a dropout, missing data will be imputed using multiple imputation and by carrying the last observed value forward. We further will perform exploratory data analyses only in those who completed follow-up testing (ie, completer's or per protocol analysis) and in those who demonstrated good adherence (ie, attended at least $83 \%$ of sessions) and compliance. ${ }^{19}$ The data will be analysed using a mixed-factor model with time (baseline and follow-up) as a within-subjects factor and condition (intervention or active control) as a between-subjects factor on composite learning and memory performance, hippocampal, and cardiorespiratory fitness outcomes. Of note, we will require an MRI overall alpha of 0.05 (corrected for multiple comparisons) for significance for hippocampal neuroimaging outcomes. The correction for multiple comparisons will be achieved by establishing a suitable voxel cluster-level threshold through Monte Carlo simulations (using the 3dClustSim program, part of the AFNI suite of image analysis programs; http:// afni.nimh.nih.gov/afni/). Effect sizes will be expressed as partial eta-squared $\left(\eta_{p}^{2}\right)$ and Cohen's $d .^{29}$

We will examine hippocampal volume and resting-state functional connectivity outcomes as potential mediators of the effects of treadmill walking exercise training on learning and memory, consistent with the methodology of Baron and Kenny. ${ }^{60}$ This statistical mediation approach is consistent with the proposed gold standard approach for examining exercise-related mechanisms of cognitive improvement at the brain systems level in the general population. ${ }^{16}$ As preconditions of the mediation analysis, we will 
first perform correlations among group (ie, intervention or control), change in learning and memory performance, and change in hippocampal volume and resting-state functional connectivity, respectively, using Spearman's $r$ rank-order correlations $(\rho)^{61}$ to test if those outcomes are interrelated. Consistent with previous preliminary studies,${ }^{21}{ }^{23}$ we expect that treadmill walking exercise training will be associated with improvements in learning and memory and increases in hippocampal volume and functional connectivity. Then, to establish mediation, we will perform hierarchical linear regression models for evaluating each of the mediators (ie, changes in hippocampal volume and resting-state functional connectivity outcomes, respectively), separately. This will involve regressing change in learning and memory performance on group in step 1 and then adding change in the mediator in step 2. As such, we expect significant effects of group on change in learning and memory, and that the effect of group (ie, intervention or control) on change in learning and memory will be attenuated, but not reach zero, when accounting for the effects of changes in hippocampal structure and function. In other words, we expect that changes in hippocampal structure and function will be partial mediators of the effects of treadmill walking exercise training on learning and memory in persons with MS with impairments in learning new information. The effect sizes from the interaction terms from the ANOVAs and correlations will serve as effect sizes for the subsequent power analyses required for a subsequent phase III RCT.

\section{Patient and public involvement}

Patients and the public were not involved in the development of the research question, experimental design, selection of outcome measures and conduct of the study. Once the data are collected and analysed, we will send lay language newsletters summarising the results of the study to each study participant via postal mail. We further plan to disseminate the results of the study via publication in peer-reviewed journals, and will send the appropriate links to study participants once the results are published.

\section{ETHICS AND DISSEMINATION \\ Adverse events}

We verify that all persons involved in the research hold current IRB certification for the protection of human subjects. Importantly, during any and all exercise activities for the current study, we will have a minimum of two researchers present who are trained in cardiopulmonary resuscitation, automated external defibrillator, first aid and emergency procedures. Further, we will monitor participants on a daily basis for adverse events such as musculoskeletal injuries related to exercise. In the event of an adverse event, this will be reported to the UAB IRB within 48 hours, and the participant will cease participation until receiving a physician's clearance to resume any study-related activities.

Throughout the study, we will monitor and record MS relapses that may affect study participation. Importantly, we expect that the overall study relapse rate will be low (ie, $<6 \%$ for both exercise and control conditions; $27 \%$ lower for exercise conditions), based on a recent systematic review of safety of exercise training in persons with MS. ${ }^{62}$ We further will minimise the potential for relapses by only including persons with MS with relative neurological stability who are on a stable disease-modifying therapy regimen. Nevertheless, all decisions (ie, inclusion/exclusion, safety of continued participation) in the event of a relapse will be made on a patient-by-patient basis, with direct consultation with the study neurologist (JRR). We will report any relapses with the final study results. In accordance with the policies and procedures of the UAB IRB, if any harm occurs to participants as a result of the trial, treatment will be provided; however, this treatment will not be provided free of charge.

\section{Confidentiality}

This study involves several approaches to maintain confidentiality. Participant information will be coded using a study code, and study forms will not contain any individually identifying information. The study code involves only an identification (ID) number that indicates the order whereby participants enrolled in the study. For example, the first participant to enrol in the study will be ID\#001, and the second participant will be ID\#002 and so on. There will be no human-derived elements in this code (eg, initials, dates and so on). The code further will not pertain to any information on random assignment to groups. The master list linking study codes to individual identities will be maintained by the investigator on a password-protected, shared drive space on the UAB server and will not be divulged to others. Paper records will be stored in a locking file cabinet in the project coordinator's locked office in the ENRL. Electronic data will be stored on UAB computers which are firewall-protected, encrypted and password-restricted. The servers are monitored at all times for outages. Secured login IDs, granted on a need-to-know basis, further are required to access confidential information.

\section{Dissemination}

The primary study results will be made available to scientists interested in MS, neuroimaging, cognition and/or exercise as a treatment for cognitive deficits in order to avoid unintentional duplication of research. Specifically, the primary results will be disseminated via peer-reviewed publications, and replication of the protocol will be encouraged. The primary results will further be disseminated via conference presentation. The data on which the final summary statistics are based will be made available to third parties in applicable data repositories.

\section{DISCUSSION}

The current study is the first adequately powered RCT to include an exercise training stimulus (ie, treadmill walking exercise that progressively increases in duration 
and intensity) that is based on research on the acute 2627 and chronic ${ }^{2123}$ effects of exercise on cognition in persons with MS that further targets hippocampal outcomes. Importantly, this study is the first RCT of exercise training to selectively recruit persons with objective impairments in learning new information in MS. ${ }^{19}$ This is a critical methodological study component, as potential treatment effects (ie, beyond simply improving cognitive performance) of exercise on MS-related cognitive dysfunction can only be assessed if participants demonstrate cognitive impairment. The current proposal will provide the first evidence for the efficacy of treadmill walking exercise training as a potential rehabilitative approach to treat MS-related learning and memory impairment. This efficacy study will include an active control condition to account for the effects of attention and social contact associated with supervised exercise training; no previous RCTs of exercise training on cognition in MS have adopted this approach. ${ }^{19}$ We further will include cardiorespiratory fitness outcomes as a manipulation check for documenting the success of the intervention; this critical feature has been lacking in previous RCTs of exercise on cognition in MS. ${ }^{19}$ The proposed study will be among the first exercise training RCTs in any population to include blinded MRI data analyses, uniquely adding another layer of rigour for improving the study's reproducibility. Such methodological features are critical for reducing threats to internal validity (ie, type I error) and providing efficacy evidence for chronic treadmill walking exercise training as a behavioural approach for managing learning and memory dysfunction in persons with MS who have the most need.

The current early-phase RCT will provide critical information for the development of future exercise trials on learning and memory in persons with MS. If successful, the current trial will provide effect sizes for power analyses for determining appropriate sample sizes for a subsequent phase III RCT. Indeed, such a line of research will lay the foundation for approaches that can be eventually translated into community-based settings for rehabilitating learning and memory in persons with MS by examining the effects of a treadmill walking exercise training intervention on learning and memory and hippocampal neuroimaging outcomes. Although results from studies of cognitive rehabilitation are promising, such interventions are not easily adapted outside of the clinic, and further do not offer health benefits beyond improved cognition. On the other hand, exercise is a behaviour that is easily adaptable in the community and offers a myriad of physical health benefits. As such, the proposed study uniquely represents the first step in developing and optimising a potentially generalisable exercise training intervention for improving behavioural (ie, learning and memory) and brain (ie, hippocampal) outcomes among cognitively impaired persons with MS. As a whole, this line of research might result in the development of exercise training guidelines that can be adapted by patients with MS for specifically improving brain health and cognition.
Importantly, there are no guidelines for persons with MS to manage cognitive impairment in the community using any approach.

If the current RCT does not result in statistically significant treadmill walking exercise-related improvements in composite learning and memory performance, we will focus on refining the exercise stimulus for specifically improving learning and memory among memory-impaired persons with MS for informing the development of a future RCT. This could involve adjusting the modality and intensity of exercise training. Indeed, there is preliminary evidence that other exercise modalities (eg, aerobic cycle ergometry) and intensities (eg, high-intensity interval training) have resulted in improvements in learning and memory in persons with MS. ${ }^{63}{ }^{64}$ If the current treadmill walking exercise training intervention does not improve learning and memory performance, another potential approach for refining the exercise stimulus involves the inclusion of additional sensory stimuli to treadmill walking exercise (ie, treadmill walking exercise plus virtual reality) for inducing cognitive change. This approach is based, in part, on animal work that demonstrates particularly large improvements in hippocampal neurogenesis, synaptogenesis, and learning and memory performance when exercise is performed in enriched environments compared with standard environments over several weeks. ${ }^{18}$ There too is evidence in persons with traumatic brain injury whereby 4 weeks of exercise plus virtual reality improved verbal and visuospatial learning and memory. ${ }^{65}$ Alternatively, if treadmill walking exercise training does not improve cardiorespiratory fitness outcomes (ie, as a manipulation check), we plan to examine the effects of a longer intervention period (ie, 6 months) on the primary and secondary study outcomes in a subsequent RCT, as has been done in older adults. ${ }^{66}$ We do not expect this to be the case, given that the exact treadmill walking exercise training stimulus resulted in large cardiorespiratory fitness improvements in our small pilot RCT. ${ }^{28}$

Although the current RCT addresses a critical problem by applying a systematically developed exercise training intervention for improving learning and memory and hippocampal structure and function among persons with MS who present with learning and memory impairment, there are several noteworthy limitations. First, the current RCT was powered based on pilot data on the effects of treadmill walking exercise training on the primary outcomes of behavioural neuropsychological tests of learning and memory in persons with MS. This study was not powered based on pilot data on the effects of treadmill walking exercise training on structural or functional hippocampal neuroimaging outcomes (ie, the secondary study outcomes), given that pilot data are not available on the present neuroimaging endpoints in persons with MS. For the current power analyses, we operationalised the effect sizes from an aerobic exercise intervention on hippocampal neuroimaging outcomes in persons with schizophrenia as a preliminary guide for powering the 
secondary study outcomes. ${ }^{30}$ Therefore, it is unknown if the study is truly adequately powered for detecting effects of treadmill walking exercise training on hippocampal structure and functional connectivity (ie, the secondary endpoints) in persons with MS who present with impairments in learning new information. The present RCT involves a relatively short intervention period, no longterm follow-up or a comparison group of persons with MS without deficits in learning new information for possibly examining the neuroprotective effects of the intervention over time. Given that preliminary work directly supports the feasibility and preliminary efficacy of 3 months of treadmill walking exercise training on learning and memory and hippocampal neuroimaging outcomes, the current adequately powered RCT seeks to rigorously examine the potential treatment effects of that exact intervention on learning and memory and its potential neural correlate(s). We believe this to be a necessary step prior to investigating the durability and sustainability of progressive treadmill walking exercise training on those outcomes in subsequent large-scale studies. We note that 3 months of aerobic exercise training has resulted in improvements in learning and memory as well as hippocampal structure and function in other populations. ${ }^{3067}$ Relatedly, the present study will not examine the effectiveness of treadmill walking exercise training on the primary outcomes in a large, national sample of persons with MS. Rather, this study will advance our systematic and rigorous line of research by providing critical efficacy data in a highly controlled environment prior to the development of a subsequent RCT for examining the effectiveness of the intervention for eventual translation into a community-based programme. The present study will not involve the collection of exploratory serum biomarkers (eg, brain-derived neurotrophic factor, vascular endothelial growth factor) as possible molecular/cellular mediators of the effects of treadmill walking exercise training on behavioural (ie, neuropsychological) and brain systems (ie, hippocampal volume and functional connectivity) outcomes. If the current RCT is successful, the examination of such potential mechanisms will be a central focus of future mechanistic research efforts. Another limitation is that the study sample will not involve persons with MS who present with substantial ambulatory disability. Rather, this RCT only focuses on persons with MS who are fully ambulatory based on pilot data and safety concerns associated with treadmill walking exercise training. Thus, the results of the current RCT might not be generalisable among all persons with MS, particularly those with severe ambulatory impairment. Finally, the present study will not directly compare the effects of treadmill walking exercise training with cognitive rehabilitation as a control comparison condition on learning and memory and hippocampal neuroimaging outcomes. Instead, this study includes an active, non-aerobic exercise training control condition in order to control for the potential effects of attention and social contact normally associated with supervised exercise training for testing the primary study hypotheses.
Examinations of the comparative and combined effects of exercise training and cognitive rehabilitation on learning and memory and hippocampal neuroimaging outcomes will be performed in subsequent effectiveness trials.

Regardless of the study outcome, we plan to communicate the trial results via peer-reviewed publications. If successful, the results from this study will eventually inform RCTs for developing rehabilitation interventions (ie, treadmill walking exercise training) for improving learning and memory and its relationship with hippocampal outcomes in a large sample of cognitively impaired persons with MS. In the long term, the results from this early-phase RCT will lay the groundwork for ultimately providing clinicians and patients with guidelines for better using chronic treadmill walking exercise for improving cognition and brain health. Such an evidencebased approach for rehabilitation, using chronic exercise training, is paramount considering the highly prevalent, disabling and poorly managed nature of MS-related learning and memory impairment.

\section{Author affiliations}

${ }^{1}$ Department of Physical Therapy, University of Alabama at Birmingham,

Birmingham, Alabama, USA

${ }^{2}$ Department of Cell, Developmental and Integrative Biology, University of Alabama at Birmingham, Birmingham, Alabama, USA

${ }^{3}$ Department of Medicine, University of Alabama at Birmingham, Birmingham, Alabama, USA

${ }^{4}$ Department of Biostatistics, University of Alabama at Birmingham, Birmingham, Alabama, USA

${ }^{5}$ Department of Radiology, University of Alabama at Birmingham, Birmingham, Alabama, USA

${ }^{6}$ Department of Neurology, University of Alabama at Birmingham, Birmingham, Alabama, USA

${ }^{7}$ Kessler Foundation, Neuropsychology and Neuroscience Research, West Orange, New Jersey, USA

Contributors BMS: study concept and design, study registration, study principal investigator, obtained IRB approval, drafting of the manuscript, critical revision of the manuscript. RWM, MBa, GRC, MBo, JRR, GRW, HG, JDL: study concept and design, critical revision of the manuscript.

Funding This paper was supported by an investigator-initiated grant from EMD Serono (One Technology Place, Rockland, Massachusetts). The principal investigator (BMS) will have access to all data from the study, complete freedom to direct the data analyses and reporting without influence of sponsors, and affirm that there is no editorial direction or censorship from sponsors.

Competing interests None declared.

Patient consent for publication Not required.

Ethics approval The study has been approved by the University of Alabama at Birmingham Institutional Review Board.

Provenance and peer review Not commissioned; externally peer reviewed.

Open access This is an open access article distributed in accordance with the Creative Commons Attribution Non Commercial (CC BY-NC 4.0) license, which permits others to distribute, remix, adapt, build upon this work non-commercially, and license their derivative works on different terms, provided the original work is properly cited, appropriate credit is given, any changes made indicated, and the use is non-commercial. See: http://creativecommons.org/licenses/by-nc/4.0/.

\section{REFERENCES}

1. Chiaravalloti ND, DeLuca J. Cognitive impairment in multiple sclerosis. Lancet Neurol 2008;7:1139-51. 
2. Geurts JJ, Bo L, Roosendaal SD, et al. Extensive hippocampal demyelination in multiple sclerosis. J Neuropathol Exp Neurol 2007;66:819-27.

3. Benedict RH, Ramasamy D, Munschauer F, et al. Memory impairment in multiple sclerosis: correlation with deep grey matter and mesia temporal atrophy. J Neurol Neurosurg Psychiatry 2009;80:201-6.

4. Hulst HE, Schoonheim MM, Van Geest Q, et al. Memory impairment in multiple sclerosis: relevance of hippocampal activation and hippocampal connectivity. Mult Scler 2015;21:1705-12.

5. Feinstein A, Magalhaes S, Richard JF, et al. The link between multiple sclerosis and depression. Nat Rev Neurol 2014;10:507-17.

6. Morrow SA, Drake A, Zivadinov R, et al. Predicting loss of employment over three years in multiple sclerosis: clinically meaningful cognitive decline. Clin Neuropsychol 2010;24:1131-45.

7. Ruet A, Deloire M, Hamel D, et al. Cognitive impairment, healthrelated quality of life and vocational status at early stages of multiple sclerosis: a 7-year longitudinal study. J Neurol 2013;260:776-84.

8. Amato MP, Ponziani G, Siracusa G, et al. Cognitive dysfunction in early-onset multiple sclerosis: a reappraisal after 10 years. Arch Neurol 2001;58:1602-6.

9. Amato MP, Langdon D, Montalban X, et al. Treatment of cognitive impairment in multiple sclerosis: position paper. J Neurol 2013;260:1452-68.

10. Rosti-Otajarvi EM, Hamalainen PI. Neuropsychological rehabilitation for multiple sclerosis. Cochrane Database Syst Rev 2014;2:CD009131.

11. Chiaravalloti ND, Wylie G, Leavitt V, et al. Increased cerebral activation after behavioral treatment for memory deficits in MS. $J$ Neurol 2012;259:1337-46.

12. Chiaravalloti ND, Moore NB, Nikelshpur OM, et al. An RCT to treat learning impairment in multiple sclerosis: the MEMREHAB trial. Neurology 2013;81:2066-72.

13. Goverover Y, Chiaravalloti ND, O'Brien AR, et al. Evidenced-based cognitive rehabilitation for persons with multiple sclerosis: an updated review of the literature from 2007 to 2016. Arch Phys Med Rehabil 2018;99:390-407.

14. das Nair R, Martin KJ, Lincoln NB. Memory rehabilitation for people with multiple sclerosis. Cochrane Database Syst Rev 2016;3:CD008754.

15. Motl RW, Sandroff BM, Benedict RH. Cognitive dysfunction and multiple sclerosis: developing a rationale for considering the efficacy of exercise training. Mult Scler 2011;17:1034-40.

16. Stillman CM, Cohen J, Lehman ME, et al. Mediators of physical activity on neurocognitive function: a review at multiple levels of analysis. Front Hum Neurosci 2016;10:1-17.

17. Vivar $\mathrm{C}$, van Praag $\mathrm{H}$. Running changes the brain: the long and the short of It. Physiology 2017;32:410-24.

18. van Praag H. Neurogenesis and exercise: past and future directions. Neuromolecular Med 2008;10:128-40.

19. Sandroff BM, Motl RW, Scudder MR, et al. Systematic, evidencebased review of exercise, physical activity, and physical fitness effects on cognition in persons with multiple sclerosis. Neuropsychol Rev 2016;26:271-94.

20. Motl RW, Pilutti LA, Hubbard EA, et al. Cardiorespiratory fitness and its association with thalamic, hippocampal, and basal ganglia volumes in multiple sclerosis. Neuroimage Clin 2015;7:661-6.

21. Leavitt VM, Cirnigliaro C, Cohen A, et al. Aerobic exercise increases hippocampal volume and improves memory in multiple sclerosis: preliminary findings. Neurocase 2014;20:695-7.

22. Sandroff BM. Exercise and cognition in multiple sclerosis: the importance of acute exercise for developing better interventions. Neurosci Biobehav Rev 2015;59:173-83.

23. Sandroff BM, Johnson CL, Motl RW. Exercise training effects on memory and hippocampal viscoelasticity in multiple sclerosis: a novel application of magnetic resonance elastography. Neuroradiology 2017;59:61-7.

24. Chan AW, Tetzlaff JM, Altman DG, et al. SPIRIT 2013 statement: defining standard protocol items for clinical trials. Ann Intern Med 2013;158:200-7.

25. American College of Sports Medicine. ACSM's resource manual for guidelines for exercise testing and prescription, seventh edition. Philadelphia, PA: Lippincott Williams \& Wilkins, 2013.

26. Sandroff $\mathrm{BM}$, Hillman $\mathrm{CH}$, Benedict $\mathrm{RH}$, et al. Acute effects of walking, cycling, and yoga exercise on cognition in persons with relapsing-remitting multiple sclerosis without impaired cognitive processing speed. J Clin Exp Neuropsychol 2015;37:209-19.

27. Sandroff $B M$, Hillman $\mathrm{CH}$, Benedict $\mathrm{RH}$, et al. Acute effects of varying intensities of treadmill walking exercise on inhibitory control in persons with multiple sclerosis: a pilot investigation. Physiol Behav 2016;154:20-7.
28. Sandroff BM, Balto JM, Klaren RE, et al. Systematically developed pilot randomized controlled trial of exercise and cognition in persons with multiple sclerosis. Neurocase 2016;22:443-50.

29. Cohen J. Statistical power analysis for the behavioral sciences. 2nd edn. Hillsdale, NJ: Lawrence Erlbaum Associates, 1988

30. Pajonk FG, Wobrock T, Gruber O, et al. Hippocampal plasticity in response to exercise in schizophrenia. Arch Gen Psychiatry 2010;67:133-43.

31. McDonald WI, Compston A, Edan G, et al. Recommended diagnostic criteria for multiple sclerosis: guidelines from the International Panel on the diagnosis of multiple sclerosis. Ann Neurol 2001;50:121-7.

32. Kurtzke JF. Rating neurologic impairment in multiple sclerosis: an expanded disability status scale (EDSS). Neurology 1983;33:1444-52.

33. Chiaravalloti ND, Balzano J, Moore NB, et al. The Open-Trial Selective Reminding Test (OT-SRT) as a tool for the assessment of learning and memory. Clin Neuropsychol 2009;23:231-54.

34. Thomas S, Reading J, Shephard RJ. Revision of the Physical Activity Readiness Questionnaire (PAR-Q). Can J Sport Sci 1992;17:338-45.

35. Motl RW, Bollaert RE, Sandroff BM. Validation of the Godin LeisureTime Exercise Questionnaire classification coding system using accelerometry in multiple sclerosis. Rehabil Psychol 2018;63:77-82.

36. Delis DC, Kramer JH, Kaplan E, et al. California Verbal Learning Test. 2nd edn (CVLT-II). San Antonio, TX: Psychological Corporation, 2000.

37. Buschke H, Fuld PA. Evaluating storage, retention, and retrieval in disordered memory and learning. Neurology 1974;24:1019-25.

38. Benedict RHB. The Brief Visuospatial Memory Test Revised (BVMT-R). Lutz, FL: Psychosocial Assessment Resources, 1997.

39. Rao S. A manual for the brief repeatable battery of neuropsychological tests in multiple sclerosis. Milwaukee, WI: Medical College of Wisconsin, 1990.

40. Strober L, Englert J, Munschauer F, et al. Sensitivity of conventional memory tests in multiple sclerosis: comparing the Rao Brief Repeatable Neuropsychological Battery and the Minimal Assessment of Cognitive Function in MS. Mult Scler 2009;15:1077-84.

41. Amato MP, Goretti B, Viterbo RG, et al. Computer-assisted rehabilitation of attention in patients with multiple sclerosis: results of a randomized, double-blind trial. Mult Scler 2014;20:91-8.

42. Mattioli F, Stampatori C, Bellomi F, et al. A RCT comparing specific intensive cognitive training to aspecific psychological intervention in RRMS: the SMICT study. Front Neurol 2014;5:1-8.

43. Boringa JB, Lazeron $\mathrm{RH}$, Reuling IE, et al. The brief repeatable battery of neuropsychological tests: normative values allow application in multiple sclerosis clinical practice. Mult Scler 2001;7:263-7.

44. Parmenter BA, Testa SM, Schretlen DJ, et al. The utility of regressionbased norms in interpreting the minimal assessment of cognitive function in multiple sclerosis (MACFIMS). J Int Neuropsychol Soc 2010;16:6-16.

45. van Geest $Q$, Hulst HE, Meijer KA, et al. The importance of hippocampal dynamic connectivity in explaining memory function in multiple sclerosis. Brain Behav 2018;8:e00954.

46. Smith DV, Utevsky AV, Bland AR, et al. Characterizing individual differences in functional connectivity using dual-regression and seed-based approaches. Neuroimage 2014;95:1-12.

47. Colcombe SJ, Kramer AF, Erickson KI, et al. Cardiovascular fitness, cortical plasticity, and aging. Proc Natl Acad Sci U S A 2004;101:3316-21.

48. Macko RF, Katzel LI, Yataco A, et al. Low-velocity graded treadmill stress testing in hemiparetic stroke patients. Stroke 1997;28:988-92.

49. Motl RW, Fernhall B. Accurate prediction of cardiorespiratory fitness using cycle ergometry in minimally disabled persons with relapsingremitting multiple sclerosis. Arch Phys Med Rehabil 2012;93:490-5.

50. Benedict RH, Fischer JS, Archibald CJ, et al. Minimal neuropsychological assessment of MS patients: a consensus approach. Clin Neuropsychol 2002;16:381-97.

51. Smith A. Symbol digit modalities test: manual. Los Angeles, CA: Western Psychological Services, 1982.

52. Gronwall DM. Paced auditory serial-addition task: a measure of recovery from concussion. Percept Mot Skills 1977;44:367-73.

53. Delis DC, Kaplan E, Kramer JH. Delis-Kaplan executive function system: technical manual. San Antonio, TX: Psychological Corporation, 2001.

54. Benton AL, Sivan AB, Hamsher K, et al. Contributions to neuropsychological assessment. Second Edition. New York, NY: Oxford University Press, 1994.

55. Patti F, De Stefano M, Lavorgna L, et al. Lesion load may predict long-term cognitive dysfunction in multiple sclerosis patients. PLoS One 2015;10:e0120754

56. Borg G. Borg's perceived exertion and pain scales. Champaign, IL: Human Kinetics, 1998. 
57. National Multiple Sclerosis Society. Stretching for people with MS: an illustrated manual. New York, NY: National Multiple Sclerosis Society, 2004.

58. Sandroff BM, Bollaert RE, Pilutti LA, et al. Multimodal exercise training in multiple sclerosis: a randomized controlled trial in persons with substantial mobility disability. Contemp Clin Trials 2017:61:39-47.

59. Bandura A. Health promotion by social cognitive means. Health Educ Behav 2004;31:143-64.

60. Baron RM, Kenny DA. The moderator-mediator variable distinction in social psychological research: conceptual, strategic, and statistical considerations. J Pers Soc Psychol 1986;51:1173-82.

61. Rousselet GA, Pernet CR. Improving standards in brain-behavior correlation analyses. Front Hum Neurosci 2012;6:119.

62. Pilutti LA, Platta ME, Motl RW, et al. The safety of exercise training in multiple sclerosis: a systematic review. J Neurol Sci 2014;343:3-7.
63. Briken S, Gold SM, Patra S, et al. Effects of exercise on fitness and cognition in progressive MS: a randomized, controlled pilot trial. Mult Scler 2014;20:382-90.

64. Zimmer P, Bloch W, Schenk A, et al. High-intensity interval exercise improves cognitive performance and reduces matrix metalloproteinases-2 serum levels in persons with multiple sclerosis: A randomized controlled trial. Mult Scler 2018;24:1635-44.

65. Grealy MA, Johnson DA, Rushton SK. Improving cognitive function after brain injury: the use of exercise and virtual reality. Arch Phys Med Rehabil 1999;80:661-7.

66. Erickson KI, Voss MW, Prakash RS, et al. Exercise training increases size of hippocampus and improves memory. Proc Natl Acad Sci U S A 2011:108:3017-22.

67. Pereira AC, Huddleston DE, Brickman AM, et al. An in vivo correlate of exercise-induced neurogenesis in the adult dentate gyrus. Proc Natl Acad Sci U S A 2007;104:5638-43. 